\title{
Administración y gestión en institución de educación superior
}

\author{
Administration and management in higher education institution
}

Administração e gestão em instituição de ensino superior

\author{
Griselda García García \\ Universidad Veracruzana, México \\ grisgg69@hotmail.com
}

\section{Resumen}

Con el propósito de mejorar la eficiencia en la administración y gestión de la Facultad de Psicología Campus Poza Rica- Tuxpan se planteó como objetivo la implementación del Modelo Organizacional de Mallot, y las variables dependientes fueron todas aquellas consideradas en el área académica como son, la docencia, la investigación y la extensión, como funciones sustantivas de la Universidad Veracruzana.

La autora expresa su efectividad para cualquier institución de educación superior, ya que los resultados evidenciaron las fortalezas, oportunidades, debilidades y amenazas que se constituyeron en áreas de oportunidad concretamente en programas específicos, con acciones y metas concretas para su mejora y logro de objetivos institucionales.

Palabras Clave: Administración; Gestión; Educación Superior

\footnotetext{
${ }^{1}$ Docente de Tiempo Completo. Universidad Veracruzana. Facultad de Psicología campus Poza RicaTuxpan. Email: grisgg69@hotmail.com
} 


\section{Abstract}

In order to improve efficiency in the administration and management of the faculty of Psychology Campus Poza Rica Tuxpan, It was outlined as objective the implementation of the Organizational Model Mallot, and the dependent variables were all those considered in the academic area, such as, the teaching, research and extension, as substantive functions of the Universidad Veracruzana.

The author expresses its effectiveness for any institution of higher education, because the results showed the strengths, weaknesses, opportunities and threats that were established in areas of opportunity, concretely, in programs with specific actions and goals for improvement and achievement of institutional objectives .

Key words : Administration; Management; Higher education

\section{Resumo}

A fim de melhorar a eficiência na administração e gestão da Escola de Psicologia Campus Poza Rica Tuxpan foi levantada como objetivo a implementação do Modelo Organizacional Mallot, e as variáveis dependentes foram todos aqueles considerados na área acadêmica como eles são, o ensino, pesquisa e extensão, funciona como substantivas da Universidad Veracruzana.

O autor expressa sua eficácia para qualquer instituição de ensino superior, como os resultados mostraram os pontos fortes, pontos fracos, oportunidades e ameaças que foram estabelecidas em áreas de oportunidade especificamente em programas específicos, com ações e metas específicas de melhoria e alcance das metas corporativas.

Palavras-chave: Administração; gestão; Educação superior

Fecha recepción: Enero 2016

Fecha aceptación: Julio 2016 


\section{Introducción}

La educación superior en los tiempos actuales, representa para América Latina y para México en particular, un elemento estratégico de las nuevas condiciones económicas, políticas, culturales y científico tecnológico que en el ámbito mundial están determinando los cambios en el quehacer educativo y, por tanto, afectando la estructura organizativa en las universidades.

Formalmente el sistema de educación superior en México se caracteriza por el desarrollo de tres funciones sustantivas: docencia, investigación, difusión y extensión de la cultura. El énfasis en el ejercicio de una o varias funciones definen de manera determinante las estructuras organizativas que sostienen a las Instituciones de Educación Superior (IES) en nuestro país.

El conocimiento y comprensión de las funciones sustantivas en las instituciones de educación superior, requiere conocer las estructuras organizativas que sostienen la vida de los establecimientos.

Como señala Mallot (2001) las organizaciones son complejas -en este caso la universidad es una organización compleja- y sin dirección las organizaciones funcionan de manera impredecible, no evolucionan necesariamente para adaptarse a su ambiente. Sin intervención tienden a evolucionar de manera caótica.

Cuando se mira una organización, sea como investigador o como participante, se puede advertir desde el primer momento, que ésta no puede comprenderse solamente como un conjunto de eventos fijos o estáticos. Por consiguiente, el cambio como fenómeno organizacional debe ser visto y asumido como un estado perenne de la organización, más que como un problema o evento temporal o pasajero.

En ese sentido Malott comenta, la paradoja de cambio se refiere a la contradicción de permutación y estabilidad, dos componentes integrales del cambio. El cambio no tiene forma fija, su producto es variable.

La Facultad de Psicología campus Poza Rica-Tuxpan fundada el 20 de octubre de 1980, con trayectoria institucional de 36 años y con la finalidad de alcanzar la capacidad y competitividad académica ha desarrollado proyectos y acciones encausadas a la mejora continua, fundamentando sus acciones en los procesos de evaluación y acreditación, 
relación con otras IES para el fortalecimiento de la disciplina e impulsar la formación de profesionales altamente competentes en el mercado laboral de acuerdo a las diferentes áreas de intervención de la psicología, transformando la realidad social a partir de un trabajo conjunto con los sectores sociales, desde la formación de una conciencia crítica, reflexiva y sobretodo propositiva para atender las situaciones, problemas o demandas actuales y emergentes en la sociedad.

El presente artículo se sustenta en el Plan Nacional de Desarrollo 2013-2018; Plan Veracruzano de Desarrollo 2011-2016; Programa Sectorial de Educación 2013-2018; Plan General de Desarrollo 2025 de la Universidad Veracruzana; Plan de Trabajo Estratégico (PTE) 2013-2017. Tradición e Innovación, de la Rectora Dra. Sara Deifilia Ladrón de Guevara González el cual plantea cuatro Dimensiones transversales que son Descentralización, Responsabilidad social, Internacionalización y Sustentabilidad, las cuales permearan a los tres ejes estratégicos I. Innovación académica de calidad, II. Presencia en el entorno con pertinencia e impacto social y III. Gobierno y gestión responsables y con transparencia, los cuales tienen como finalidad orientar el destino de la Universidad Veracruzana.

\section{Método}

Se utilizó el modelo organizacional de Mallot como estratégica metodológica, iniciando con la misión y visión institucional.

Misión ${ }^{2}$. La Facultad de Psicología Región Poza Rica-Tuxpan tiene como propósito fundamental formar Licenciados en Psicología con un perfil profesional cuya premisa sea integrar conocimientos y habilidades propios de la disciplina en un marco de respeto, compromiso, apertura, colaboración, autonomía, tolerancia, equidad y honestidad que

${ }^{2}$ Misión, Visión y Valores. Desde 1999 con la puesta en marcha del Modelo Educativo Integral y Flexible MEIF) el Programa Educativo (PE) de Licenciatura en Psicología. 
posibiliten la resolución de problemáticas sociales en los distintos campos de las áreas: Clínica, Educativa, Organizacional y Social.

Visión. La Facultad de Psicología Región Poza Rica - Tuxpan. Pretende contar con un programa educativo reconocido por organismos evaluadores, flexible en su organización académica y administrativa mediante un sistema de información y gestión a partir del uso intensivo de las nuevas tecnologías, con Cuerpos Académicos consolidados que pertenezcan al Sistema Nacional de Investigadores y con egresados certificados fuertemente vinculados a los sectores sociales.

Valores. En la Facultad de Psicología el marco de referencia que inspira y regula la vida académica y administrativa, se caracteriza por valores compartidos y cultivados por sus miembros que son el respeto, responsabilidad, compromiso, honestidad, apertura, colaboración, sustentabilidad, armonía, autonomía, tolerancia, equidad y profesionalismo.

Posteriormente se describió el contexto institucional: capacidad y competitividad académica, que refiere los recursos humanos con que cuenta y cuyos indicadores nos hablan del nivel de desarrollo, compromiso, productividad y del programa educativo reconocido y acreditado.

Planta Académica. Con respecto al personal académico, lo conforman 30 personas distribuidas como lo muestra la Tabla 1.

Tabla 1. Planta académica

\begin{tabular}{|c|c|c|c|c|}
\hline \multicolumn{5}{|c|}{ Personal académico } \\
\hline Género & 24 mujeres & & 6 hombres & \\
\hline $\begin{array}{l}\text { Actividad } \\
\text { principal } \\
\text { tiempo } \\
\begin{array}{l}\text { destinado a } \\
\text { Universidad }\end{array}\end{array}$ & $\begin{array}{l}13 \text { PTC } \\
\text { Docentes } \\
\text { Tiempo } \\
\text { Completo }\end{array}$ & $\begin{array}{lr}7 & \text { Docentes } \\
\text { con horas } \\
\text { base }\end{array}$ & $\begin{array}{l}3 \text { Técnicos } \\
\text { académicos }\end{array}$ & $\begin{array}{l}7 \text { Docentes } \\
\text { interino } \\
\text { por horas }\end{array}$ \\
\hline
\end{tabular}


En cuanto a su formación académica, 16 poseen grado de Doctor, 6 candidatos a Doctor, 7 con Maestría y 1 con Licenciatura. Justo aquí se hace necesario establecer una estrategia para el relevo generacional ya que 12 de los 13 PTC tienen más de 25 años de antigüedad y sus edades oscilan entre 45 y 65 años de edad.

Cuerpos Académicos. El Programa cuenta con el Cuerpo Académico Consolidado (CAC) Educación, Salud y Evaluación; Cuerpo académico en Consolidación (CAEC) Calidad de Vida y Desarrollo Humano y el Cuerpo Académico en Formación (CAEF) Educación, Violencia y Comportamiento Sustentable. En ellos participan los docentes desarrollando las líneas de generación y aplicación del conocimiento. Las propuestas se realizan desde los órganos colegiados y desde los cuerpos académicos. Del total de docentes, 25 de ellos participan en CA, lo que posibilita su participación en investigación, dirección de tesis, en eventos académicos propios del CA al que están adscritos y tutoría en investigación.

Estos cuerpos académicos a través de sus representantes han conformado redes con sus pares ubicados al interior y al exterior de la Universidad Veracruzana. Ver tabla 2

Tabla 2. Trabajo en Redes de los CA

\begin{tabular}{|c|c|}
\hline NOMBRE DE LA RED & CUERPO ACADEMICO \\
\hline \multirow[t]{2}{*}{ Familia y desarrollo } & Consolidado UV-CA-233 \\
\hline & Educación, Salud y Evaluación \\
\hline \multirow{8}{*}{ Familia, educación, salud y trabajo. } & Consolidado UV-CA-233 \\
\hline & Educación, Salud y Evaluación \\
\hline & En Consolidación UV-CA-268 Calidad \\
\hline & de Vida y Desarrollo \\
\hline & Humano \\
\hline & En Formación UV-CA-303 \\
\hline & Educación, $\quad$ Violencia \\
\hline & Comportamiento Sustentable \\
\hline \multirow{7}{*}{$\begin{array}{l}\text { Red de CA y Grupos de } \\
\text { Investigación de la Región Sur- } \\
\text { Sureste del CNEIP }\end{array}$} & Consolidado UV-CA-233 \\
\hline & Educación, Salud y Evaluación \\
\hline & En Consolidación UV-CA-268 Calidad \\
\hline & de Vida y Desarrollo Humano \\
\hline & En Formación UV-CA-303 \\
\hline & Educación, Violencia y \\
\hline & miento Sustentable \\
\hline
\end{tabular}

Fuente. Méndez, M. (2015) Informe de labores 2014-2015 Directora.

Programa de Estímulos al desempeño del Personal Académico (PEDPA)

Respecto a la participación de académicos en el Programa de Estímulos al Desempeño del Personal Académico (PEDPA) se presentan en la Tabla 3. 
Tabla 3. Participantes PEDPA

\begin{tabular}{lll}
\hline $\begin{array}{l}\text { TIPO } \\
\text { CONTRATACION }\end{array}$ & $\begin{array}{l}\text { PARTICIPANTES } \\
\text { PEDPA }\end{array}$ \\
\hline PTC & 12 \\
TA & 1 \\
PA & 2 \\
TOTAL & 15 \\
\hline e. Méndez, M. (2015) Informe de labores 2014-2015 Directora.
\end{tabular}

Programa de Formación de Académicos (ProFA). El 95\% de los académicos adscritos a esta institución asisten con regularidad a los cursos, seminarios y diplomados que convoca el Programa de Formación de Académicos (ProFA) diseñado por el Departamento de Competencias Académicas, dependiente de la Dirección General de Desarrollo Académico de la Universidad Veracruzana, en coordinación con la Comisión Mixta de Capacitación y Adiestramiento.

PRODEP. En el caso del Programa de Desarrollo Profesional Docente (PRODEP) de 13 profesores de tiempo completo (PTC) 11 cuentan con el perfil PRODEP; 1 participa como Nuevo Personal de Tiempo Completo (NPTC); 1 PTC es de nuevo ingreso y 2 pertenecen al Sistema Nacional de Investigadores (SNI). Los profesores de tiempo completo han diversificado formalmente su carga académica: en docencia frente a grupo, tutorías, realizando proyectos de investigación o aplicación innovadora del conocimiento y participando en actividades de gestión académica.

Investigación. Si bien en los últimos tres años la producción y divulgación científica desde los cuerpos académicos fue importante, anualmente en promedio cada CA publica un libro, un capitulo de libro, 2 artículos y participan en congresos nacionales e internacionales con publicaciones de memoria en extenso con ISSN. Se considera prioritario desarrollar estrategias que permitan el fortalecimiento y promoción de la misma. Se observa la necesidad de seguir respaldando acciones encaminadas al mantenimiento de la consolidación de CA y al fortalecimiento de los CA en consolidación a fin de que transiten de ese nivel a consolidado. Además se deben aplicar estrategias que permitan el desarrollo 
a través de la obtención de recursos extraordinarios para los distintos cuerpos académicos.

Vinculación. La Facultad de Psicología realiza sus procesos de vinculación a través de proyectos registrados en el Sistema de Información para la Vinculación Universitaria (SIVU), del Modulo de Consulta Externa y de las academias por área de conocimiento en Psicología Clínica, Psicología Educativa, Psicología Organizacional, Psicología Social y comunitaria. Entre los resultados relevantes que el SIVU presenta en este periodo destacan: 18 proyectos y 9 actividades de vinculación. SIVU (2016)

La dependencia cuenta con un Modulo de Consulta Externa y el Centro de Entrenamiento y Educación Especial de Poza Rica (CEEEPoR). El primero proporciona servicios psicológicos a la comunidad universitaria y a la población en general, atendiendo en promedio 1000 pacientes por año. El segundo atiende a 40 niños con capacidades diferentes.

Si bien existen acciones efectivas dentro del apartado de vinculación se considera que es necesario atender aspectos como la formalización de las relaciones interinstitucionales a través de convenios.

Fortalecer la colaboración entre las diversas instituciones nacionales y extranjeras a fin de generar espacios para el intercambio, la movilidad y el establecimiento de redes académicas. Desarrollar estrategias dirigidas a nuestros egresados que atiendan aspectos tales como bolsa de trabajo y oferta de capacitación.

Lo anterior posibilitaría cumplir con la meta de generar recursos extraordinarios

Posgrado PNPC de CONACyT. La facultad de Psicología ha formado hasta hoy 30 generaciones de licenciados en Psicología, y es nuestra oportunidad diseñar la maestría en Psicología con base a LGAC que se trabajan en la institución, también participar en la convocatoria de CONACYT para ingresar al PNPC ya que las condiciones académica, de infraestructura, administrativa y la ausencia de un posgrado en esta disciplina en la región norte de Veracruz son propicias.

Relevo generacional. Dentro de la plantilla académica actual 12 de los 13 PTC tienen una antigüedad de más de 23 años y sus edades oscilan entre 45 y 65 años de edad.

De 14 profesores de asignatura se cuenta con cuatro académicos con Doctorado y seis con grado de maestría mismos que participan en actividades relacionadas con la gestión, 
tutorías e investigación lo que los perfila como relevos generacionales adecuados a la misión y propósito del programa educativo.

En general se puede establecer que la planta académica está en un proceso de renovación, que requiere considerar estrategias para el relevo generacional con profesionales que cumplan con las condiciones que exige la dinámica actual de la Educación Superior, nuestra disciplina y las necesidades sociales regionales.

Alumnos. La matricula atendida es de un promedio de 344 por periodo. Distribuida por género se observa predominio del género femenino que representa el $75 \%$ ante el $25 \%$ que corresponde al masculino. Referente a la edad se observa que el $75 \%$ se ubica en el rango de 20 a 24 años, el $23 \%$ representa a la población de menos de 20 años y el $2 \%$ equivale a los estudiantes mayores de 25 años.

La Universidad Veracruzana cuenta con un Sistema Institucional de Tutorías (SIT) y el programa tiene una Coordinación del Sistema Tutorial (CST). Actualmente son 24 tutores académicos, que atienden a la matrícula total de 344 alumnos.

Los alumnos tienen acceso al Programa Nacional de Becas para la Educación Superior (PRONABES), a las Becas Fundación Universidad Veracruzana y las que otorga la Universidad Veracruzana que son:

Inscripción. Condonación del pago por derechos arancelarios.

Escolares. Se otorgan a los alumnos más destacados de las diferentes entidades académicas. Deportivas. Para alumnos que en representación de la Universidad Veracruzana se distinga en eventos deportivos.

A pesar que se atiende al $100 \%$ de la población estudiantil, se observa la necesidad de fortalecer la tutoría académica y de investigación con la participación activa de los actores del proceso. Asimismo desarrollar el aspecto correspondiente a la planeación y operación del Programa de Apoyo a la Formación Integral (PAFI) que complemente la atención durante la trayectoria de los estudiantes.

Otro aspecto relacionado con los estudiantes que requiere atención es la promoción adecuada de los programas de movilidad estudiantil que se convierta en estrategia que impacte en la formación integral del estudiante. 
Programa de Calidad y Actualización del Plan de Estudios. El Consejo Nacional para la Enseñanza e Investigación en Psicología otorga la acreditación por el periodo 2014-2019 a este programa de licenciatura en psicología por cumplir con los requisitos de calidad educativa establecidos por el Comité de Acreditación del Consejo.

Desarrolla un plan de estudios basado en los lineamientos generales de operatividad del Modelo Educativo Integral y Flexible. La filosofía del MEIF plantea una propuesta de educación centrada en el alumno, y favorece el alcanzar los objetivos de una formación integral en las áreas intelectual, humana, profesional y social. Para el logro de esa formación integral, el plan se sustenta en los ejes: teórico, heurístico y axiológico. Dicha perspectiva de formación integral genera una alternativas educativa que desarrolla en el alumno tanto habilidades, conocimientos y actitudes que lo hacen más competente para las exigencias del medio socioeconómico.

A partir del 2011 la facultad inició con el proceso de evaluación del plan de estudios para dar paso al proceso de Rediseño del Plan de Estudios de la licenciatura en Psicología, actualmente se cuenta con un significativo avance del $80 \%$.

Infraestructura. Las instalaciones de la Facultad se ubica en dos espacios, uno central ubicado en la Unidad de Ciencias de la Salud específicamente en el Edificio B-CSER que posee una estructura de tres niveles, en los cuales se encuentran 8 aulas, con una capacidad promedio de 30 a 40 estudiantes. Todas ellas habilitadas con pintarrón, pizarrón de corcho, cortinas, escritorio y silla., una sala de posgrado con capacidad de 50 personas, una sala para académicos, 12 cubículos para profesores, 1 cámara de Gesell, 1 modulo de consulta externa en psicología clínica, educativa, organizacional, social y comunitaria. Existen espacios compartidos con las demás facultades adscritas a la DES Ciencias de la Salud como lo son: Aula Magna con capacidad para 90 personas; la biblioteca; el centro de computo en el cual se presta el servicio de acceso de equipos de computo; el almacén; el estacionamiento y cancha de concreto para actividades deportivas.

La DES cuenta con equipo para la red inalámbrica lo que permite previa clave el acceso de estudiantes y académicos desde cualquier punto dentro de las instalaciones.

El otro espacio que es periférico, alberga el Centro de Entrenamiento y Educación Especial de Poza Rica (CEEEPoR); destinado principalmente para actividades prácticas y servicios 
hacia la comunidad. Existe un auditorio para 120 personas, área para actividades al aire libre, recreativas, culturales y deportivas. Área para atención individualizada 22 espacios, aula para atención grupal (5 a 10 personas), aula con cámara de Gessel con capacidad para 5 a 10 personas, aula para terapia ocupacional, aula acondicionada como ludoteca, consultorio odontológico, consultorio medico, oficina general, oficina de coordinación de consulta externa, cubículo de psicología, cubículo de pedagogía, cubículo de trabajo social, una casa con cocina, sala, comedor, recamara para el aprendizaje de actividades de la vida cotidiana y un área para educación continua.

En el apartado de Infraestructura, es ineludible que la creación y modernización de espacios esté direccionada a garantizar un desarrollo físico armónico y equilibrado y en atención a las recomendaciones de los organismos evaluadores.

Financiero. Para el desarrollo de las funciones sustantivas y adjetivas la Facultad gestiona y ejerce un presupuesto proveniente de recursos ordinarios y extraordinarios.

Tabla 4. Proyectos de financiamiento

\begin{tabular}{lll}
\hline Proyectos & Montos & Otorgante \\
\hline 811 & $\$ 240,150.00$ & Universidad Veracruzana \\
131 & $\$ 84,958.24$ & Cuotas de recuperación \\
132 & $\$ 1,666,680.23$ & Aportaciones de alumnos \\
\hline \multicolumn{2}{l}{ Fuente. Méndez, M. (2015) Informe de labores 2014-2015 Directora. }
\end{tabular}

La situación de escasez de recursos es un reto para la inteligencia y creatividad. Si ofertamos servicios como educación continua, los CA y la investigación accedan a la obtención de recursos extraordinarios como la participación en programas federales y estatales. Los centros de extensión como el CEEEPoR y los módulos de consulta externa que realicen un servicio autofinanciado. El comité pro-mejoras que realice un programa activo con padres de familia; egresados; sector empresarial y asociación civil y fundaciones.

Normatividad. Existe un Reglamento Interno que requiere armonizarse y ser aprobado por el H. Consejo Universitario General 


\section{Resultados}

Se identificaron las fortalezas, oportunidades, debilidades y amenazas, siguientes:

\section{Fortalezas:}

- Programa educativo acreditado por CNEIP organismo reconocido por COPAES.

- Plan de estudios a nivel licenciatura con un currículo basado en los lineamientos de un modelo educativo integral y flexible.

- Sistema Institucional de Tutorías dirigido a los estudiantes.

- Programa de actividades de extensión para difundir la carrera.

- Un porcentaje significativo de los académicos de la Facultad tiene formación de posgrado.

- Personal con experiencia académica en el área de su especialidad, preparación profesional y experiencia laboral.

- Presencia de sistema de evaluación de desempeño académico de carácter obligatorio y periódico.

- Organización a través de academias de conocimiento y módulos de consulta externa en sus áreas clínica, educativa, organizacional y social.

- Cuerpo académico consolidado

- Dos Cuerpos Académicos en consolidación con probabilidades de transitar a un nivel de Consolidado en un futuro cercano.

- Presencia de proyectos de vinculación con el sector productivo, educativo y social.

- Infraestructura y equipamiento básico para el desarrollo de las actividades académicas.

- Existencia de un Presupuesto permanente.

Oportunidades:

- Prestigio de la Facultad al ser reconocida como una institución acreditada para formar Psicólogos.

- Incremento en la demanda de estudios de la Licenciatura en Psicología por lo que se podría ampliar la matrícula.

- Ingresar recursos extraordinarios a través de Educación Continua, misma que ha probado tener demanda en esta región y concretamente en esa disciplina. 
- Ingresar recursos en especie o a través de cuotas de recuperación por servicios de capacitación a empresas, instituciones o grupos.

- Capitalizar la infraestructura, misma que podría ser útil en fines de semana y periodos intersemestrales con la oferta de servicios formativos, capacitación y educación continua a la comunidad en la región.

- Mantener permanentemente el vínculo con egresados ahora profesionistas económicamente activos que podrían contribuir con sus saberes así como a continuar su formación en posgrado. Esto hace que nuestra facultad fortalezca su pertinencia social.

- El CEEEPoR podría consolidarse con un programa certificado de servicios, ampliar su cobertura de manera organizada y planeada dirigida a los diversos niveles educativos, con estudios permanentes de Orientación educativa, vocacional y hacia el trabajo. A través de cuotas de recuperación que posibiliten su autofinanciamiento con transparencia.

- El modulo de consulta externa en área clínica podría consolidar sus servicios especializados, certificados y realizar peritajes a través de un programa eficiente y con transparencia.

- Diseñar y ofertar la maestría en psicología. Se cuenta con docentes calificados y egresados con aspiraciones formativas y en la región no hay oferta.

- Captación de recursos extraordinarios a través de la investigación y CA

- Impulsar a los estudiantes a la movilidad nacional e internacional

\section{Debilidades:}

- Reducido numero de académicos hablan un segundo idioma.

- Inexistencia de un programa de posgrado ofertado por la dependencia. que permitiría a los egresados continuar con su formación profesional.

- Insuficiente captación de recursos externos para investigación. Ausencia de convenios con instituciones

- Escasa movilidad de los estudiantes y académicos

- No se ingresan recursos extraordinarios a través de educación continua, misma que ha probado tener demanda en esta región y concretamente en esa disciplina. 
- No se ingresan recursos en especie o a través de cuotas de recuperación por servicios de capacitación a empresas, instituciones o grupos.

- Se subutiliza la infraestructura, misma que podría ser útil en fines de semana y periodos intersemestrales con la oferta de servicios formativos, capacitación y educación continua a la comunidad en la región.

- No se mantiene el vínculo con egresados ahora profesionistas económicamente activos y que podrían contribuir con sus saberes así como a continuar su formación en posgrado. Esto hace que nuestra facultad fortalezca su pertinencia social.

\section{Amenazas:}

- Políticas en educación superior.

- Reducción del presupuesto a nivel nacional y estatal para la educación superior.

- Aumento del número de universidades privadas de bajo costo y calidad.

- Apertura de la carrera de psicología en otras instituciones ubicadas en la región.

Derivado de lo antes citado se diseñó la siguiente propuesta de mejora continua que esta integrada en tres ejes rectores y metas.

\section{EJE I. Innovación académica de calidad}

Meta. 1.1. Reconocimiento de calidad del Programa educativo de la facultad.

El Programa Educativo (PE) de Psicología se someterá a evaluaciones internas y externas para retroalimentarlo y generar procesos de actualización permanente, en base a los principios del Modelo Educativo.

Se realizará la Re-Acreditación en 2019 del PE para asegurar la matrícula de calidad en la licenciatura y se orientará a la facultad hacia la Acreditación internacional.

Elaborar y mejorar los manuales de organización de procedimientos específicos al interior de la facultad con apego a la normatividad universitaria.

Se diseñara el PE de la Maestría en Psicología y participará en la evaluación de CONACYT para obtener el reconocimiento en el PNPC como programa de calidad.

Se concluirá con el nuevo diseño del Plan de Estudios de la licenciatura en Psicología para responder a las condiciones del entorno laboral y social, promoviendo la transversalización de los criterios de sustentabilidad, equidad, congruencia y pertinencia social.

Fomentar entre los estudiantes la continuación del estudio de otros idiomas o dar continuidad al inglés para impulsar la movilidad estudiantil. 
Consolidar la creación e impartición de PAFIS que permitan apoyar a estudiantes en situación de riesgo en EE que presenten altos índices de reprobación o con bajo rendimiento escolar.

Promover mediante la tutoría el egreso en tiempo mínimo de periodos, mediante una adecuada asesoría para el desarrollo óptimo de su trayectoria escolar, promoviendo así la eficiencia terminal.

Impulsar a través de las academias el trabajo colegiado y el desarrollo de procesos de evaluación estandarizados de las Experiencias Educativas, así como unificar criterios y contenidos que sean actualizados para mantener la pertinencia del PE, de tal manera que se posibilite el desarrollo de las competencias que requiere el Licenciado en Psicología para su adecuada intervención profesional.

Impulsar la impartición de EE de manera virtual utilizando los recursos tecnológicos institucionales para apoyar la trasversalización de las competencias desarrolladas mediante el área de formación básica.

Incluir como EE las practicas profesionales en el rediseño del plan de estudios, para fortalecer la formación de nuestros estudiantes y armonizarlo con PTE 2014-2017

Meta 1.2 . Incrementar el número Perfil Deseable PROMEP con los nuevos PTC

Meta 1.3 Atracción y retención de estudiantes

Meta 1.4 Lograr que los CAs incrementen la producción académica de calidad durante cada ciclo anual, mediante la investigación socialmente pertinente

EJE II. Presencia en el entorno con pertinencia social

Meta 2.1. Lograr que un $40 \%$ de egresados mantengan comunicación con la facultad y se incorporen a la bolsa de trabajo de la UV.

Meta 2.2 Desarrollar al menos un evento académico científico local al año y un evento nacional cada cinco años.

Meta 2.3 Actualizar el plan estratégico de vinculación y realizar al menos 2 convenios con instituciones de los sectores público, productivo o social para la realización de servicio social y prácticas escolares.

Meta 2.4 Elaborar un programa de trabajo enfocado a promover la equidad de género y la interculturalidad a través de proyectos de intervención e investigación social.

EJE III. Gobierno y gestión responsables y con transparencia 
3.1 Hacia el 2017, al menos el 50\% de los convenios que se hayan formalizado generarán recursos financieros para la institución.

\section{Conclusión.}

El Modelo organizacional de Mallot permitió identificar el contexto institucional, en el que se destacan los elementos que orientan las acciones relacionadas con la capacidad, competitividad académica y su compromiso con los diversos sectores.

Se identificaron las funciones sustantivas de la Facultad de Psicología, como de sus integrantes. Los resultados posibilitaron una propuesta centralizada en el estudiante como eje rector y señala explícitamente que a él debe dedicarse la mayoría de los esfuerzos de ésta institución. Finalmente se propone generar los acuerdos necesarios entre los diversos actores, para impulsar los cambios cualitativos que debe proyectar la Facultad de Psicología Campus Poza Rica-Tuxpan de la Universidad Veracruzana.

\section{Bibliografía}

DOF (2013). Plan Nacional de Desarrollo 2013-2018. Presidencia de la República. Recuperado de http://pnd.gob.mx/

Gobierno del Estado de Veracruz (2011). Plan Veracruzano de Desarrollo 2011 - 2016. Recuperado de http://portal.veracruz.gob.mx/pls/portal/docs/PAGE/STP/TRANSPARENCIA/PVD/ P VD\%202005-2010.PDF.

Gobierno del Estado de Veracruz (2011). Plan Veracruzano de Desarrollo 2011-2016. Programa Veracruzano de Educación. Recuperado de http://www.veracruz.gob.mx/programadegobierno/servicio/pvd/ http://www.veracruz.gob.mx/finanzas/files/2012/08/tf07-ps-pvd-11-16-pve-4.pdf

Malott E. M. (2001) Paradoja del cambio organizacional. México: Trillas.

Méndez, M. (2015). Informe de labores 2014-2015. Facultad de Psicología. Directora. Recuperado de http://www.uv.mx/pozarica/psicologia/files/2016/05/PsicologiaInformeSep2014Ago s2015.pdf 
SEP (2013). Programa Sectorial de Educación 2013-2018. Secretaria de Educación Pública. Recuperado de http://www.dof.gob.mx/nota_detalle_popup.php?codigo=5326569

UV (2013). Programa de Trabajo Estratégico 2013-2017. Presentación al H. Consejo Universitario General 16 de dic. 2013. Recuperado de http://www.uv.mx/pte20132017/files/2013/12/presentacion-PTE-2013-2017.pdf

UV (2016). Universidad Veracruzana Plan General de Desarrollo 2025. Recuperado de http://www.uv.mx/planeacioninstitucional/documentos/documents/PlanGeneraldeD esarrollo2025.pdf 